Article

\title{
Icaritin Reduces Oral Squamous Cell Carcinoma Progression via the Inhibition of STAT3 Signaling
}

\author{
Jian-Guang Yang ${ }^{1}$, Rui Lu ${ }^{1,2}$, Xiao-Jing Ye ${ }^{1}$, Jing Zhang ${ }^{1,2}$, Ya-Qin Tan ${ }^{1}$ and Gang Zhou ${ }^{1,2, *}$ \\ 1 The State Key Laboratory Breeding Base of Basic Science of Stomatology (Hubei-MOST) and \\ Key Laboratory of Oral Biomedicine Ministry of Education, School and Hospital of Stomatology, \\ Wuhan University, Wuhan 430079, China; 2014103040017@whu.edu.cn (J.-G.Y.); \\ KQ000874@whu.edu.cn (R.L.); 2014103040018@whu.edu.cn (X.-J.Y.); zhang_lin_ling@whu.edu.cn (J.Z.); \\ 2015103040008@whu.edu.cn (Y.-Q.T.) \\ 2 Department of Oral Medicine, School and Hospital of Stomatology, Wuhan University, Wuhan 430079, China \\ * Correspondence: gordonzhou@tom.com; Tel.: +86-27-8768-6213
}

Academic Editor: Terrence Piva

Received: 21 November 2016; Accepted: 6 January 2017; Published: 12 January 2017

\begin{abstract}
Icaritin, a traditional Chinese medicine, possesses antitumor activity. The current study aimed to investigate icaritin effect and potential mechanism on oral squamous cell carcinoma (OSCC) development. OSCC cells proliferation, apoptosis, and autophagy were analyzed after incubation with icaritin at different concentrations and incubation times. The expressions of proteins related to proliferation, apoptosis, and autophagy, as well as signal transducer and activator of transcription 3 (STAT3) signal network, were also evaluated by western blot. Furthermore, STAT3 was knocked down by siRNA transfection to determine STAT3 role in OSCC cell proliferation and apoptosis. An oral specific carcinogenesis mouse model was used to explore icaritin effect on OSCC in vivo. Icaritin significantly inhibited OSCC proliferation in vitro and reduced the expression of both the cell-cycle progression proteins cyclin A2 and cyclin D1. Besides, icaritin increased cleaved caspase 3 and cleaved poly-(ADP-ribose) polymerase expression leading to apoptosis, and it activated autophagy. Icaritin significantly inhibited the expression of phospho-STAT3 (p-STAT3) in a dose- and time-dependent manner. In the in vivo experiment, the number of malignant tumors in the icaritin-treated group was significantly lower than the control. Overall, icaritin suppressed proliferation, promoted apoptosis and autophagy, and inhibited STAT3 signaling in OSCC in vitro and in vivo. In conclusion, icaritin might be a potential therapeutic agent against OSCC development.
\end{abstract}

Keywords: icaritin; oral squamous cell carcinoma; signal transducer and activator of transcription 3; premalignant lesion

\section{Introduction}

Oral squamous cell carcinoma (OSCC), as one of the most common cancers in the world, is a major public health problem [1]. OSCC worldwide incidence exceeds 300,000 cases and about 145,000 of them die annually [2]. Despite recent therapeutic advances in oral cancer, the mortality and over five-year survival rates remain unchanged, which can be largely attributed to the local recurrences and distant metastasis [3,4]. During the multistep carcinogenesis, the oral cavity exhibits clinically defined premalignant lesions such as oral leukoplakia and erythroplakia [5]. The premalignant lesions are believed to have high risk associated with the progression to cancer [6]. Thus, targeting these lesions may represent a good option to prevent tumor progression and ultimately reduce the incidence of OSCC. Therefore, new and effective therapeutic agents guaranteeing safety and minimal toxicity are urgently required for OSCC prevention and treatment. 
Icaritin is a hydrolytic product of icarin from Epimedium Genus, a traditional Chinese herbal medicine [7] (Figure 1). Previous studies showed that icaritin has various pharmacological and biological effects, including cardiovascular function improvement, hormone regulation, and modulation of immunological function [8]. In addition, it induces apoptosis and growth inhibition, downregulates tumor angiogenesis, and inhibits invasion and epithelial-to-mesenchymal transition in human cancer cells, such as prostate carcinoma cells, endometrial cancer cells, breast cancer stem/progenitor cells, hematological malignancy cells, renal cell carcinoma cells, lung cancer cells, and multiple myeloma cells [9-17].<smiles>COc1ccc(-c2oc3c(CC=C(C)C)c(O)cc(O)c3c(=O)c2O)cc1</smiles>

Figure 1. Chemical structure of icaritin.

Recent studies revealed that signal transducer and activator of transcription 3 (STAT3) is constitutively activated and involved in the process of apoptosis resistance in many malignant tumors [18-21]. STAT3 is also well-known for its role in cancer-related inflammation, immunity, and autophagy [22,23]. Janus kinases JAKs phosphorylate gp130, leading to the recruitment and activation of STAT3, which then result in STAT3-mediated transcriptional regulation [18,22]. Some reports suggested that OSCC tumor cells frequently produce high levels of p-STAT3 [18,20], and various JAKs and STAT3 inhibitors could abrogate OSCC growth [19,24-27]. In addition, icaritin exerted an anti-tumor effect via inhibiting JAKs/STAT3 activation $[12,14,16]$. Thus, on the basis of this evidence, we hypothesized that the inhibition of JAK2/STAT3 aberrant expression induced by icaritin might be a potential therapeutic strategy against OSCC development.

In the present study, we investigated the effect of icaritin on OSCC cell line growth and tried to elucidate its potential molecular mechanisms for the first time. We also used an oral specific carcinogenesis mouse model to observe the ability of icaritin to prevent OSCC development in vivo.

\section{Results}

\subsection{Icaritin Inhibited Proliferation of OSCC Cells}

To explore the anti-tumor effects of icaritin on OSCC cells, dose and time -response studies were performed in CAL27 and SCC9 cells. The results showed that icaritin significantly inhibited cell proliferation compared with the control group $(p<0.05)$ (Figure 2A). In particular, icaritin treatment at the concentrations of 8 and $16 \mu \mathrm{M}$ resulted in a more efficient cell growth inhibition at 48 and $72 \mathrm{~h}$, compared with the inhibition at $24 \mathrm{~h}$ (Figure 2B). Icaritin $\mathrm{IC}_{50}$ value on CAL27 or SCC9 cells were 15.99 or $10.36 \mu \mathrm{M}(24 \mathrm{~h}), 11.87$ or $6.11 \mu \mathrm{M}(48 \mathrm{~h})$, and 9.93 or $3.95 \mu \mathrm{M}(72 \mathrm{~h})$, respectively. Western blot results showed that icaritin treatment on CAL27 and SCC9 cells reduced the expression of both the cell-cycle progression proteins cyclin A2 and cyclin D1 in a dose-dependent manner (Figure 2C).

\subsection{Icaritin Induced Apoptosis of OSCC Cells}

Cell apoptosis was remarkably increased in both icaritin-treated CAL27 and SCC9 cells. Annexin-V positive cells were significantly increased in the icaritin treated groups $(4,8$, and $16 \mu \mathrm{M})$ compared with control group $(p<0.05)$ (Figure 3A,B). Icaritin increased the expression of 
both apoptosis-related cleaved caspase 3 and cleaved poly-(ADP-ribose) polymerase (PARP) in a dose-dependent manner (Figure 3C).

A

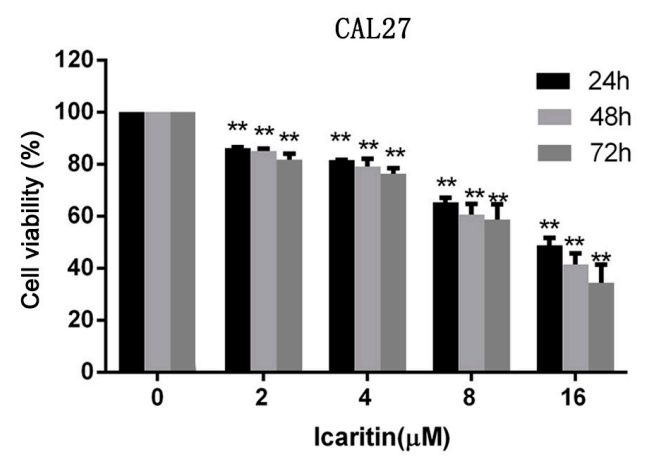

B

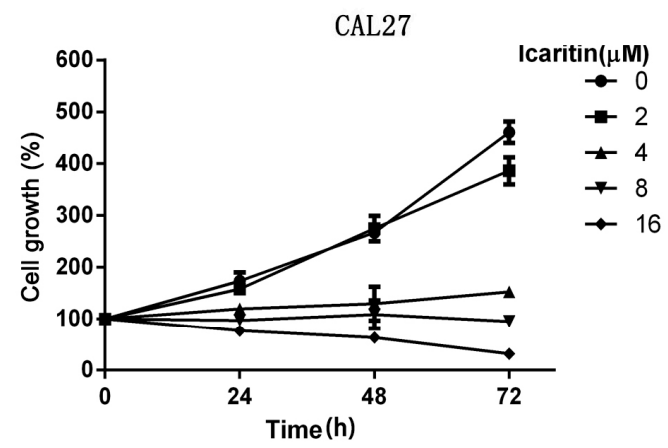

$\mathrm{C}$

CAL27
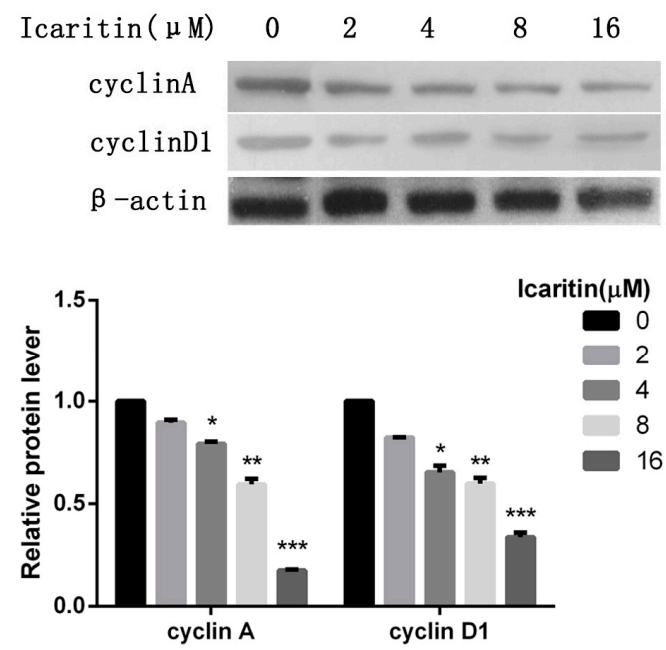

$\operatorname{scC} 9$

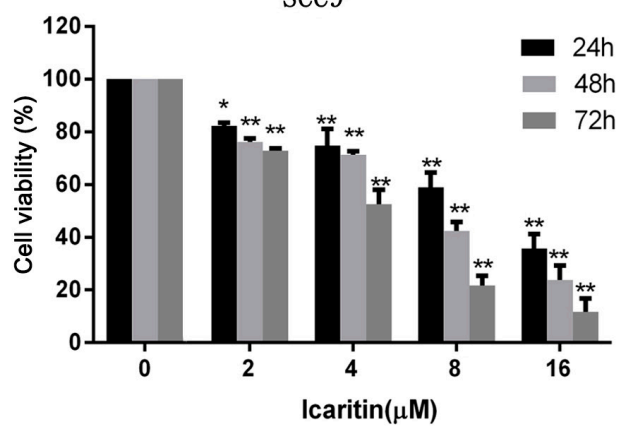

$\operatorname{SCC} 9$

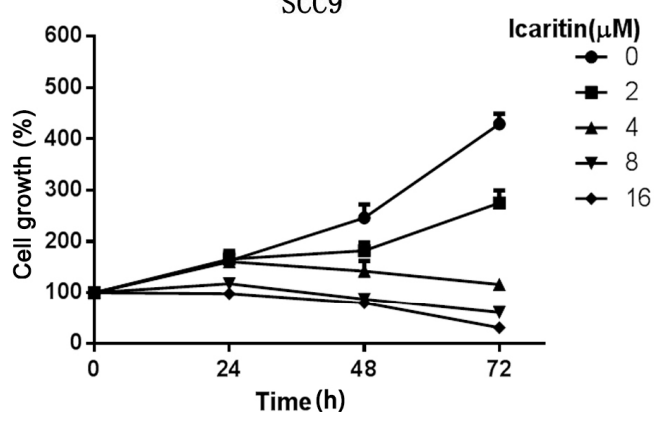

SCC9
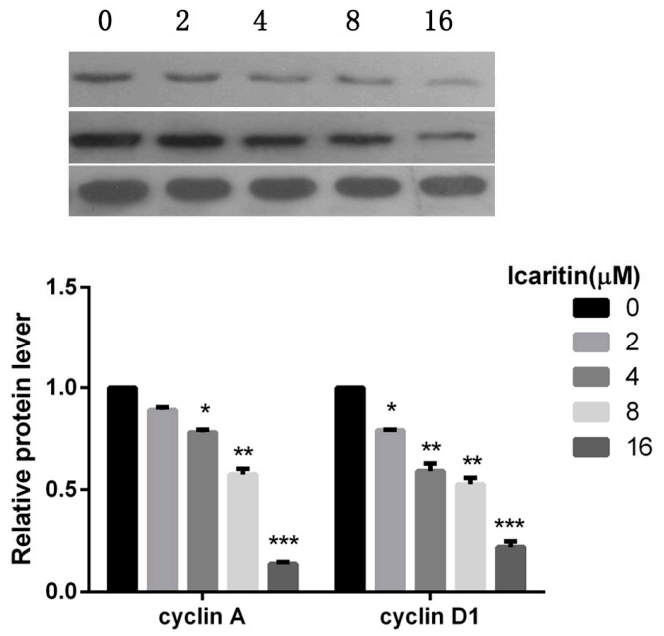

Figure 2. Icaritin inhibited OSCC cells proliferation. (A,B) Dose and time-response curves showing icaritin effects on OSCC cell lines growth inhibition. CAL27 and SCC9 cells were treated with icaritin at increasing concentrations $(2,4,8$, and $16 \mu \mathrm{M})$ for 24,48 , and $72 \mathrm{~h}$. Cell viability was evaluated by CCK-8 assay. Data were expressed as mean \pm standard deviation (SD) $\left(n=3\right.$, in triplicate). ${ }^{*} p<0.05$; and ${ }^{* *} p<0.01$ versus $0 \mu \mathrm{M}$ group; (C) Icaritin downregulated cyclin A and cyclin D1 expression, as evaluated by western blot. $\beta$-actin was used as a loading control. Relative density data of each protein was quantified by $\beta$-actin using Image J, and the data represented the mean of three independent experiments. ${ }^{*} p<0.05$; ${ }^{* *} p<0.01$; and ${ }^{* * *} p<0.001$ versus $0 \mu \mathrm{M}$ group. 
A
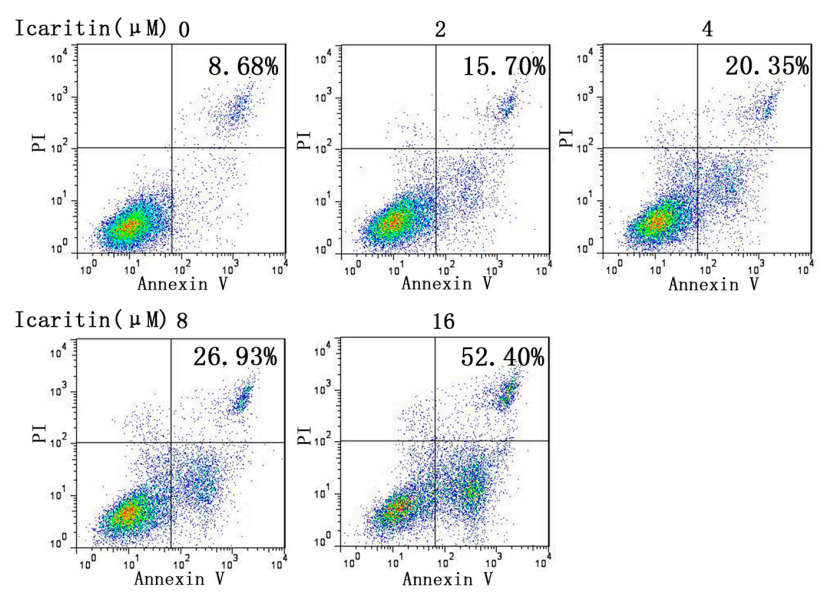

B
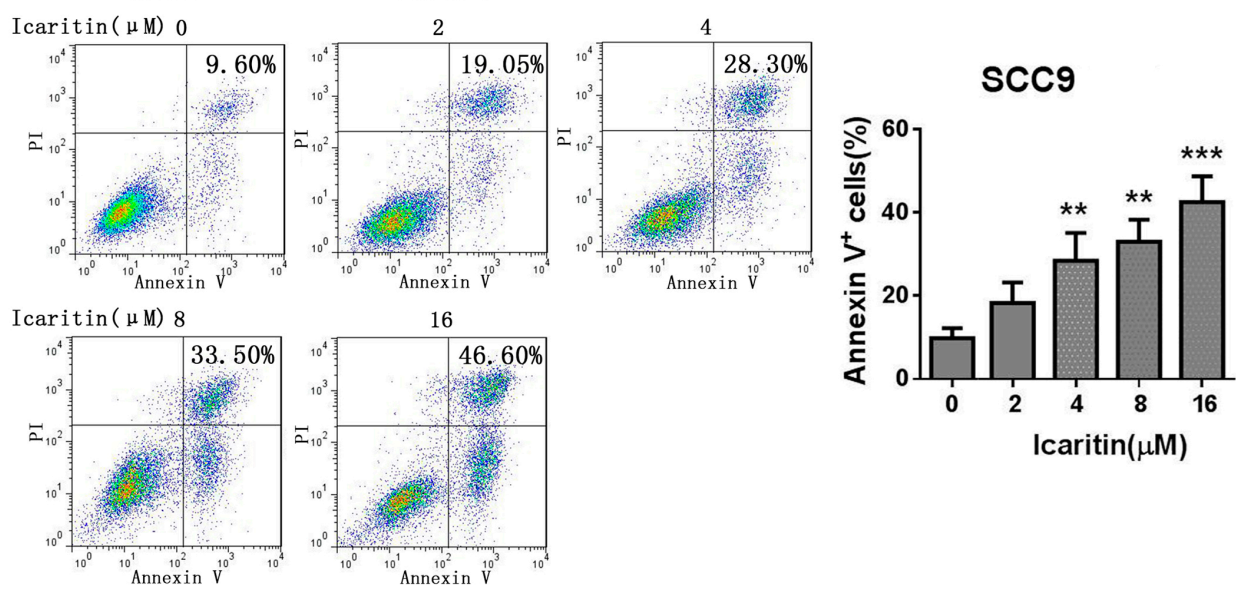

$\mathrm{C}$
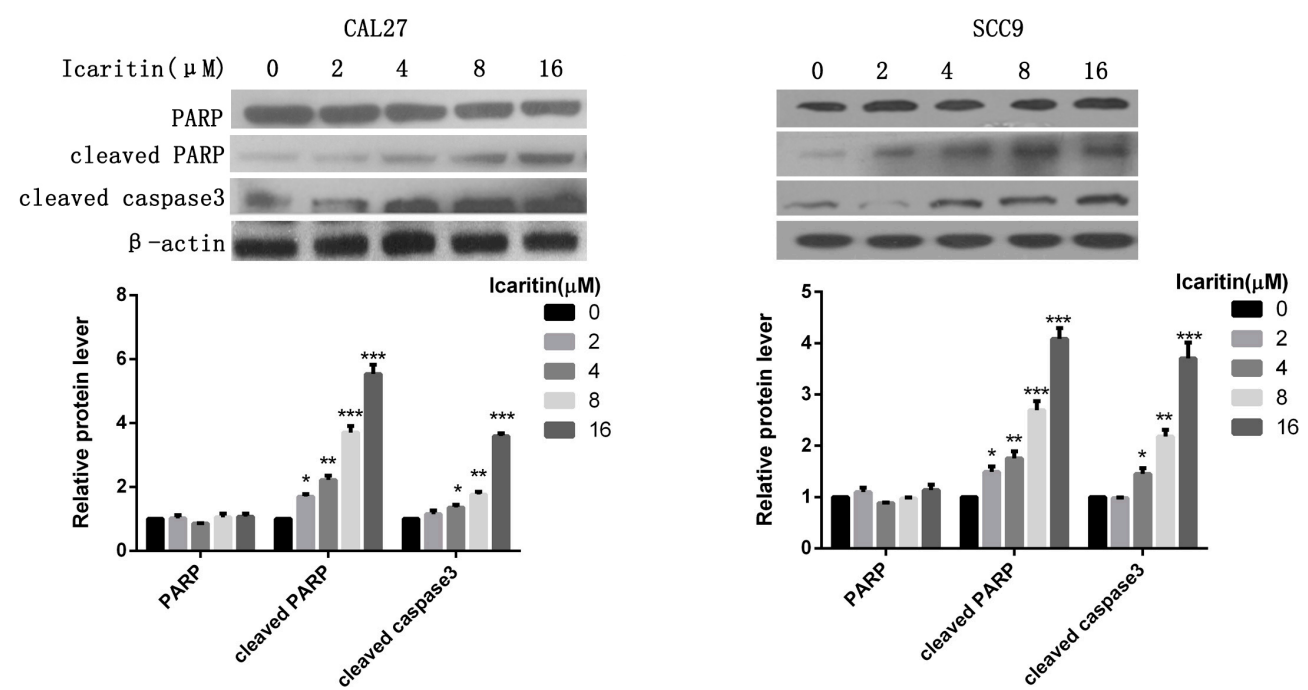

Figure 3. Icaritin induced apoptosis in OSCC cells. (A,B) OSCC cell apoptosis after icaritin treatment. CAL27 and SCC9 cells were treated with icaritin at indicated doses for $48 \mathrm{~h}$, then were harvested and stained with Annexin-V FITC and PI. Annexin-V positive cells were measured by flow cytometry. Columns represent the average percent of Annexin V positive cells. Data were expressed as mean $\pm \mathrm{SD}$ $\left(n=3\right.$, in triplicate). ${ }^{*} p<0.05 ;{ }^{* *} p<0.01,{ }^{* * *} p<0.001$. Representative images were shown in the left panel; (C) Effects of icaritin on apoptosis related proteins, total and cleaved PARP, and cleaved caspase 3. $\beta$-actin was used as a loading control. Relative density data of each protein was quantified by $\beta$-actin using Image J, and the data represented the mean of three independent experiments. ${ }^{*} p<0.05$; ${ }^{* *} p<0.01$; and ${ }^{* * *} p<0.001$ versus $0 \mu \mathrm{M}$ group. 


\subsection{Icaritin Triggered Autophagy in OSCC Cells}

As shown in Figure 4A, punctated fluorescence indicating autophagic vacuoles was significantly increased in a dose-dependent manner in the cytoplasm of icaritin treated OSCC cells. Consistently, western blot results demonstrated that icaritin could induce the conversion of LC3I to LC3II, indicative of autophagic activity, in CAL27 and SCC9cells, increase BECN1 expression, and reduce p62/SQSTM1 expression, both of them autophagy-associate protein (Figure 4B).

A
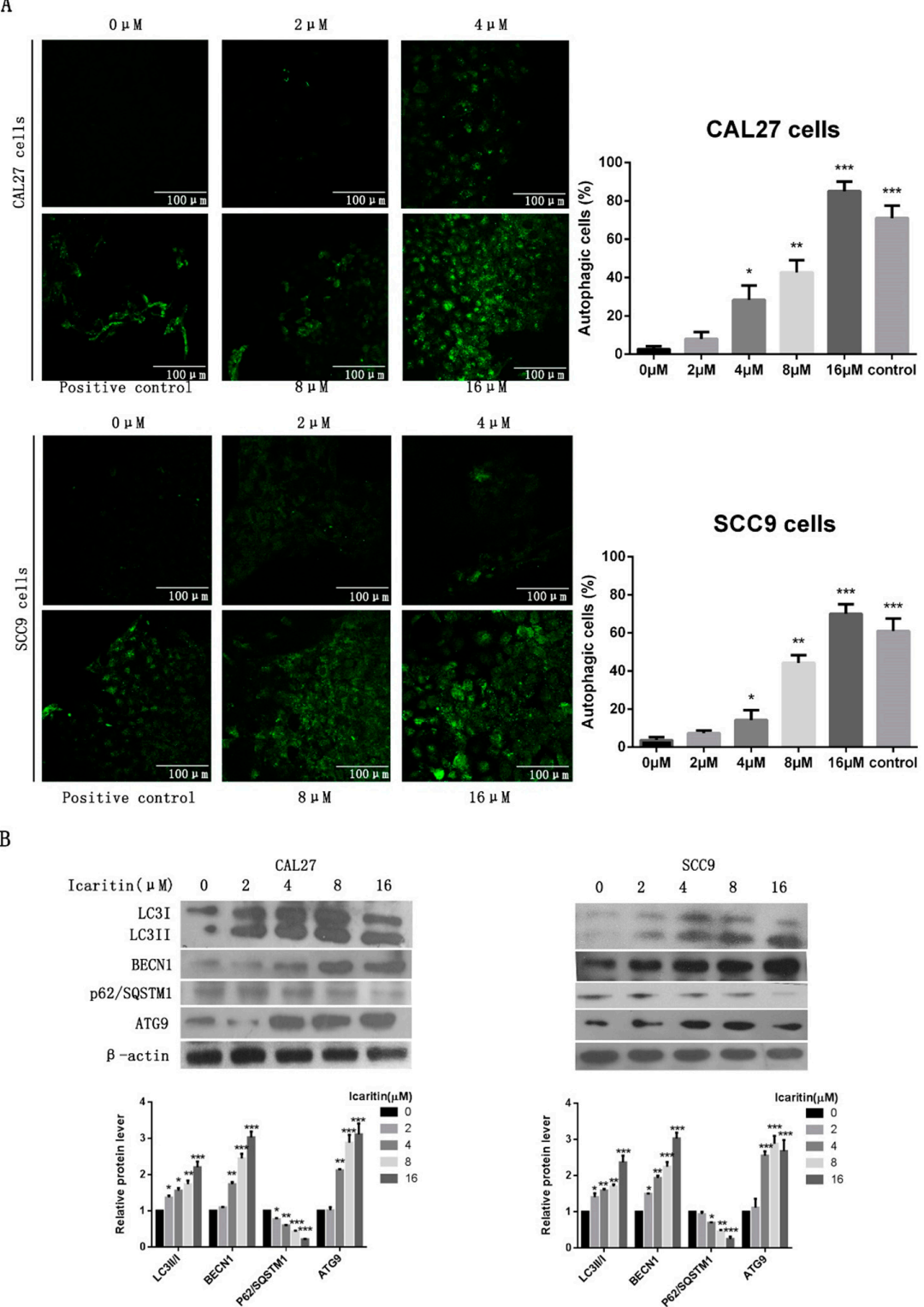

Figure 4. Icaritin triggered OSCC cell autophagy. (A) Representative images of MDC staining of CAL27 and SCC 9 cells after icaritin treatment at the indicated concentration for $48 \mathrm{~h}$. Punctated fluorescence in the cytoplasm indicated the formation of autophagic vacuoles. Magnification: $\times 400$ for MDC staining. ${ }^{*} p<0.05$; ${ }^{* *} p<0.01$; and ${ }^{* * *} p<0.001$ versus $0 \mu \mathrm{M}$ group; (B) CAL27 and SCC9cells were treated with icaritin at different concentration for $48 \mathrm{~h}$, and then LC3I/II, BECN1, p62/SQSTM1, and ATG9 were detected by western blot analysis. $\beta$-actin was used as a loading control. Relative density data of each protein was quantified by $\beta$-actin using Image $\mathrm{J}$, and the data represented the mean of three independent experiments. ${ }^{*} p<0.05 ;{ }^{* *} p<0.01$; and ${ }^{* *} p<0.001$ versus $0 \mu \mathrm{M}$ group. 


\subsection{Icaritin Inhibited JAK2/STAT3 Signaling in OSCC Cells}

To elucidate icaritin anti-tumor mechanisms on OSCC cells, several major oncogenic signaling pathways - such as JAK2, STAT3, and ERK—-were examined. The results demonstrated that icaritin significantly inhibited the expression of phospho-STAT3 (p-STAT3) in a dose- and time-dependent manner, although it had no effects on total STAT3 protein levels (Figure 5A,B). Phospho-JAK2 (p-JAK2) was also inhibited by icaritin (Figure 5A,B). Besides, p-ERK1/2 level was increased in CAL27 cells (Figure 5A).

A
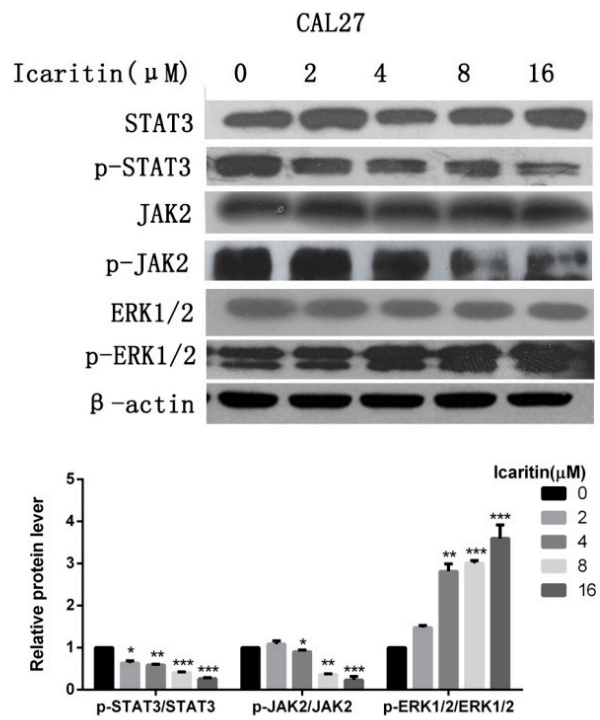

SCC9

$\begin{array}{lllll}0 & 2 & 4 & 8 & 16\end{array}$
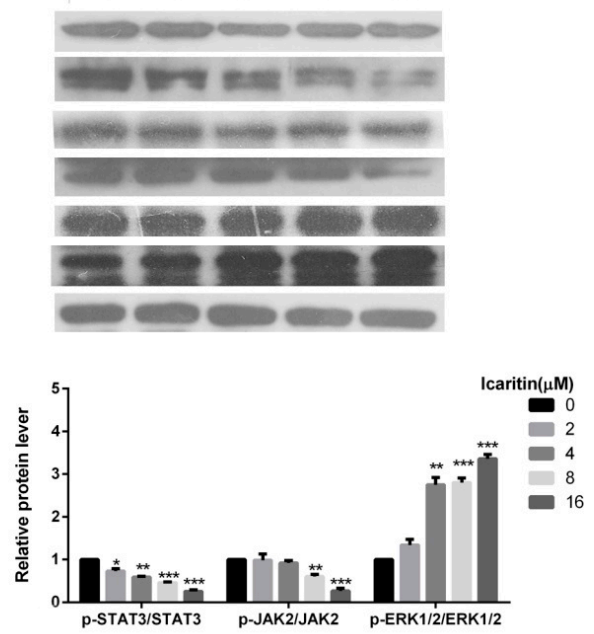

SCC9
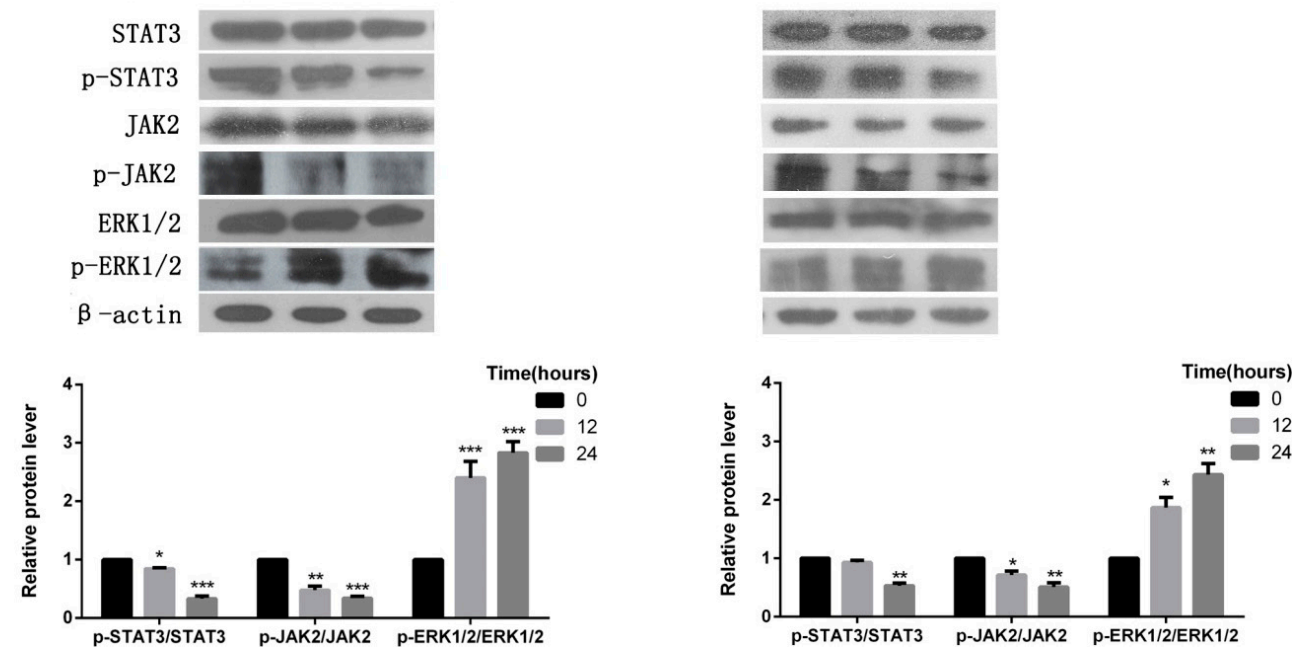

Figure 5. Effects of icaritin on oncogenic signaling pathways in OSCC cells. (A) CAL27 and SCC9 cells were treated with icaritin at the indicated doses for $24 \mathrm{~h}$. Protein levels were detected by western blot using the correspondent antibodies. $\beta$-actin was used as a loading control. Relative density data of each protein was quantified by $\beta$-actin using Image J, and the data represented the mean of three independent experiments. ${ }^{*} p<0.05$; ${ }^{* *} p<0.01$; and ${ }^{* * *} p<0.001$ versus $0 \mu \mathrm{M}$ group. Icaritin inhibited the expression of p-JAK2 and p-STAT3, while p-ERK1/2 expression was upregulated; (B) CAL27 and SCC9 cells were treated with icaritin at the indicated interval times. p-JAK2 and p-STAT3 inhibition was increased with time lasting. ${ }^{*} p<0.05 ;{ }^{* *} p<0.01$; and ${ }^{* * *} p<0.001$ versus 0 h group. 
2.5. Icaritin-Induced Proliferation Inhibition and Apoptosis Were Regulated by STAT3 Signaling in OSCC Cells

To further investigate whether STAT3 signaling activity directly affects the biological effects of icaritin in OSCC cells, CAL27 cells were transfected with siRNA against STAT3 or a control vector (nonspecific siRNA), and the successful transfecttion was confirmed by western blot analysis (Figure 6A). Cyclin A2 and p62/SQSTM1 expressions were clearly decreased after transfection, while cleaved caspase 3 expression increased (Figure 6A). The effect of icaritin in the inhibition of CAL27 cells proliferation was significantly enhanced $(p<0.05)$ (Figure 6B), and icaritin-induced apoptosis in STAT3 siRNA group was significantly increased compared with apoptosis in the nonspecific siRNA group $(p<0.01)$ (Figure 6C). These results indicated that icaritin inhibited cell growth and induced apoptosis of OSCC cell lines in vitro through STAT3 signaling.

A
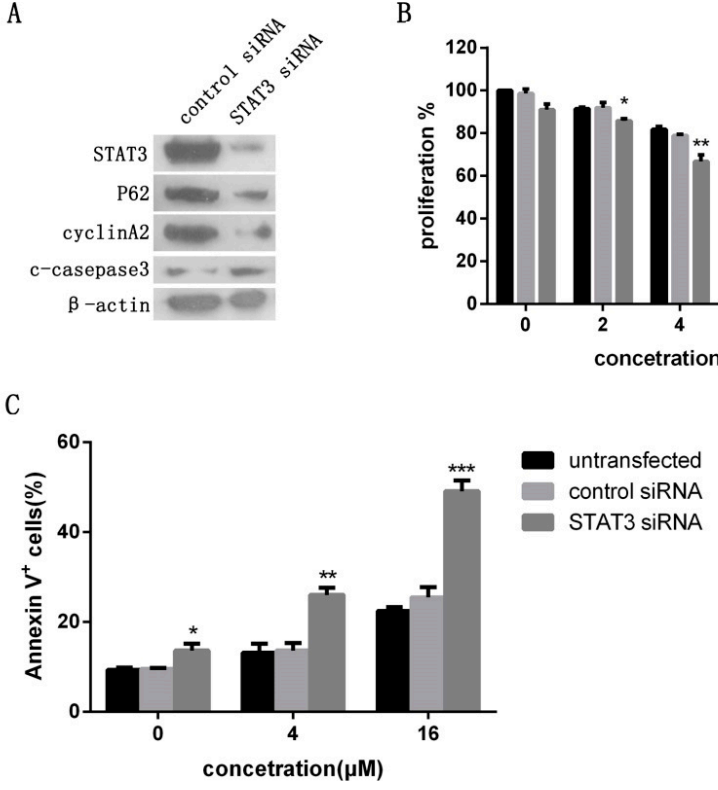

B

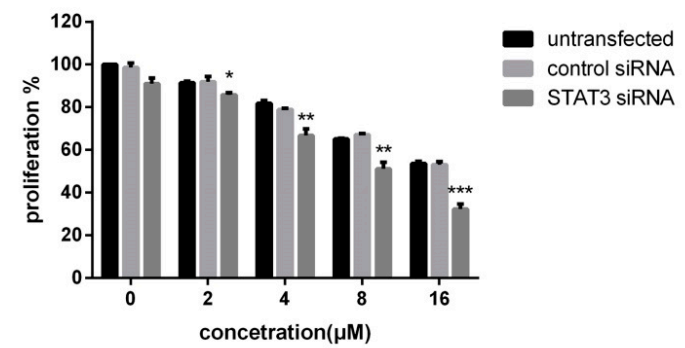

Figure 6. Antitumor effect of icaritin was affected by the levels of STAT3 activity in OSCC cells. (A) CAL27 cells were transfected with STAT3 and non-specific siRNA, and the successful transfection was confirmed by western blotting. STAT3 knockdown affected the expression of proliferation and apoptotic proteins; $(\mathbf{B}, \mathbf{C})$ STAT3 silencing enhanced the effects of icaritin on CAL27 cell growth inhibition and apoptosis promotion. CAL27 cells were transfected with either non-specific siRNA or STAT3 siRNA, and then treated with icaritin at the indicated doses for $48 \mathrm{~h}$. Cell proliferation and apoptosis were detected. Data were expressed as mean $\pm \operatorname{SD}\left(n=3\right.$, in triplicate). ${ }^{*} p<0.05 ;{ }^{* *} p<0.01$; and ${ }^{* * *} p<0.001$ versus $0 \mu \mathrm{M}$ group.

\subsection{Icaritin Inhibited Oral Tumorigenesis Induced by $4 N Q O$ and Potential Premalignant Lesions Progression} In Vivo

Next, icaritin effect on OSCC progression in vivo was assessed. As show in Figure 7A, the size of the lesions became smaller after a period of eight weeks of treatment with icaritin compared to the beginning of the treatment. In contrast, at week 22, the lesions in the control group showed a large size compared with the location at week 14. Microscopically, most tumors in the icaritin untreated mice developed atypical squamous epithelial cells. The number of malignant tumors in the icaritin-treated group was significantly less than the control group $(p<0.05)$ (Figure $7 \mathrm{C})$. Icaritin also reduced the total number of tumor lesions, although this difference was not statistically significant (Figure 7B). Besides, icaritin treatment did not affect the weight of the mice. At the end of experiment (the 22nd week), the mean body weight was $30.49 \mathrm{~g} \pm 1.40 \mathrm{~g}$ in icaritin-treated groups, which was not significantly different from the weight at the beginning of the treatment (the 14 th week, $31.03 \mathrm{~g} \pm 1.33 \mathrm{~g}, p>0.05$ ) 
(Figure 7D). Furthermore, icaritin treatment clearly reduced the expression of p-STAT3 and p-JAK2 in the epithelium of the tumor compared with untreated control (Figure 7E).

A

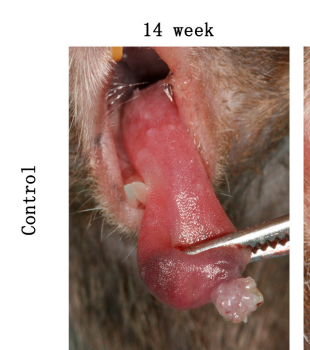

22 week
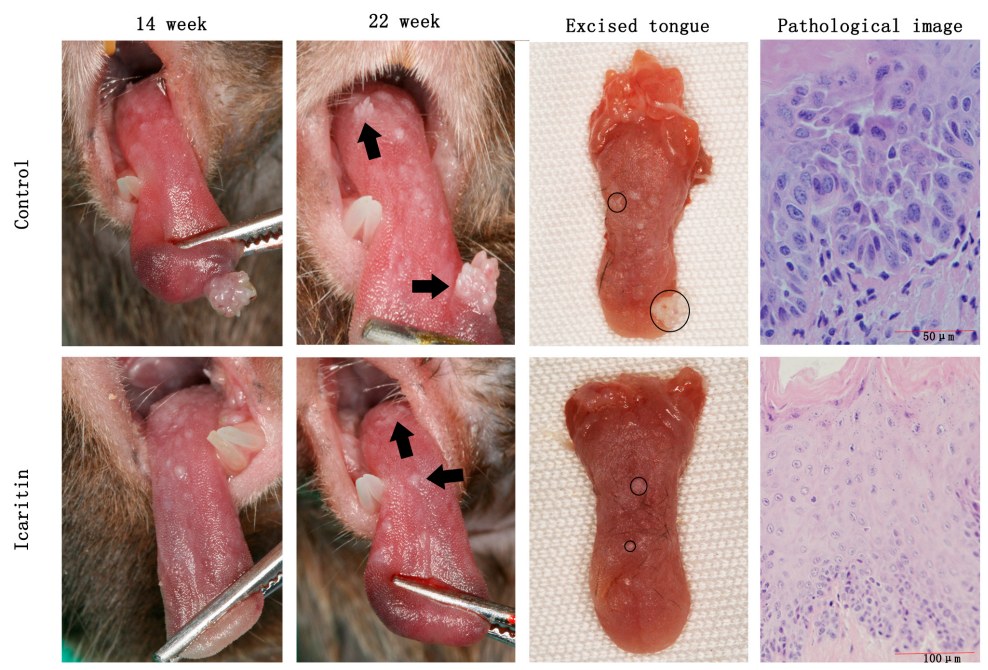

B

C
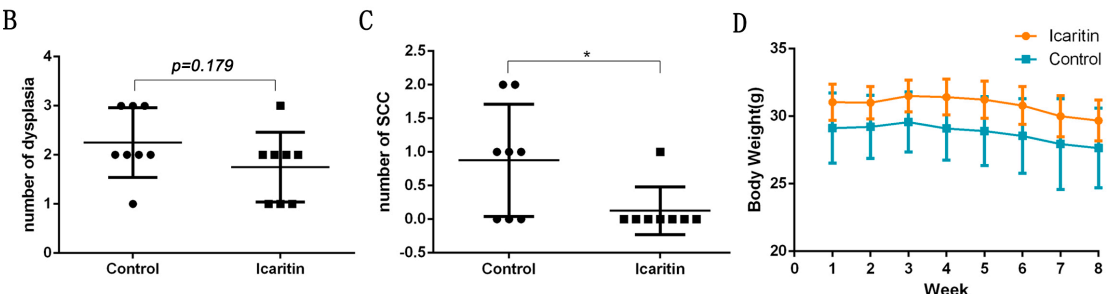

$\mathrm{E}$
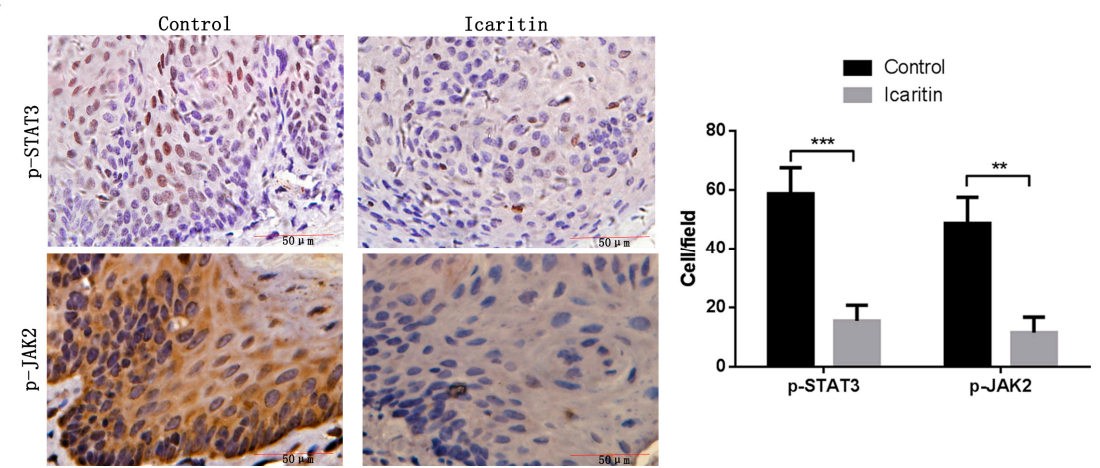

Figure 7. Icaritin decreased the number of oral tumors and prevented the malignant conversion of premalignant lesions. (A) At week 22, the lesions in the control group showed a significantly large size compared with the location at week 14 (black arrow). Under higher magnification, the lesions revealed atypical epithelial cells with squamous differentiation and basement membrane partially disappeared. In the icaritin-treated group, the lesion size became smaller (black arrow). Under higher magnification, the epithelium was clearly hyperplastic, while the cells displayed no evident atypia. The lesions on the excised tongue were marked with black circle; (B) The total number of lesions (benign and malignant tumors) in the icaritin treated group was less than that in control group, although no significant difference was observed; (C) The number of malignant tumors was significantly less in the icaritin-treated mice compared to the untreated group. ${ }^{*} p<0.05$ versus control group; (D) Mice body weight was measured every week during the experiments. Data were expressed as mean \pm SD; (E) Immunohistochemical analysis of p-STAT3 and p-JAK2 ( $\times 400$ of magnification). Icaritin significantly inhibited the expression of p-STAT3 and p-JAK2 in the epithelium of the tumor. ** $p<0.01$; and ${ }^{* * *} p<0.001$ versus control group. 


\section{Discussion}

Recently, a new approach in cancer chemoprevention is the exploration of the important pharmacological action of many natural products such as lycopene, curcumin, epigallocatechin-3-gallate (EGCG) and resveratrol, as well as icaritin [14]. Previous studies demonstrated that cell-cycle progression is dynamically and strictly regulated by cyclins, which are critical for normal cell cycle progression $[10,28]$. Tong et al. indicated that icaritin-induced cell growth inhibition was due to the decrease of cyclin D1 in human endometrial cancer cells [10]. Zhu et al. reported that icaritin reduced cyclin A, cyclin B, and CDK2 expression in multiple myeloma [14]. Our study showed that icaritin clearly reduced cyclin D1 and cyclin A2 expression in a dose-dependent manner. These results suggested that icaritin could regulate cell-cycle related effectors. Tong et al. also observed that icaritin induced apoptosis of Hec1A cells, but the proportion of necrotic cells was not significantly increased, which was consistent with our results [10]. Thus, this result could indicate that growth inhibition exerted by icaritin might be due to apoptosis induction. Apoptosis, a form of regulated cell death, is promoted by the activation of cysteine proteases of the caspase 3 [29]. PARP play a role in DNA repair, while cleaved PARP is considered as a marker of apoptosis and could be induced by caspase 3 [30]. In the present study, we found that icaritin treatment resulted in a significant increment of cleaved caspase- 3 and PARP in a dose-dependent manner. Based on the above, these results suggested that icaritin-induced OSCC cell apoptosis was due, at least in part, to the caspase pathway activation.

STAT3 plays a key role in various biological processes, including tumor cell proliferation promotion, survival, tumor invasion, angiogenesis, autophagy, and immunosuppression [21,22,31]. Some studies have indicated the important role of STAT3 in the progression of OSCC $[18,32,33]$. TGF- $\alpha$ /EGFR autocrine or paracrine activation is frequently observed in OSCC, and STAT3 is a vital signaling network downstream of TGF- $\alpha$ /EGFR [34]. In primary tumors of OSCC patients, STAT3 is constitutively activated and induces expression of cyclin D1, which is associated with cancer cell growth [21]. STAT3 activation also leads to increased cell proliferation, angiogenesis, and multidrug-resistance, as well as reduced cell apoptosis, thus promoting tumorigenesis in OSCC $[22,35,36]$. In the present study, icaritin clearly inhibited STAT3 activity in OSCC cell lines, associated with upstream p-JAK2 inhibition in a dose- and time-dependent manner. Furthermore, icaritin suppressed the expression of p-JAK2 and p-STAT3 in mice carcinoma tissue, which was consistent with our results in vitro. In addition, STAT3 knockdown enhanced the effects of icaritin on CAL27 cell growth arrest. Finally, cyclin D1, a downstream target of STAT3-regulated genes [18], was significantly reduced in STAT3 knockdown CAL27 cells. In contrast, knockdown of STAT3 in CAL27 cells significantly increased the expression of cleaved-caspase 3. Consistently with these results, Zhu et al. found that silencing of STAT3 not only resulted in a remarkable cell proliferation decreases, but also induced increased apoptosis in icaritin-treated multiple myeloma cells compared with cells treated with nonspecific siRNA [14]. These results suggested that the anti-tumor effects of icaritin were mainly through STAT3 signaling inhibition in OSCC.

Previous studies demonstrated that icaritin could also suppress cancers through other signaling pathways, such as ERK, cell cycle-regulated, and apoptosis signals $[10,11,15,16,37]$. Previous reports show that icaritin induced ERK1/2 sustained phosphorylation and activation, thus inhibiting the growth of endometrial and breast cancer cells [10,11]. In the present study, icaritin also increased p-ERK1/2 expression in CAL27 cells. In addition, icaritin could induce cell growth inhibition of prostate and lung cancer cells through cell cycle arrest or apoptosis signaling $[9,13]$. Our initial results showed that icaritin treatment reduced cyclin A2 and cyclin D1, while it activated cleaved-PARP and cleaved-caspase 3 . Therefore, besides JAK2/STAT3 signaling, ERK, cell cycle and apoptosis signal pathways might be also involved with icaritin treatment in OSCC.

Many stress pathways elicit autophagy and apoptosis simultaneously within the same cell. Autophagy induction is exacerbated when apoptosis is suppressed [38]. Some studies have reported that autophagy and apoptosis could be sequentially induced by anti-tumor drugs in cancer cells $[39,40]$. 
In the present study, the results indicated that icaritin induced autophagy in a dose-dependent manner. As far as we know, this is the first report showing the effects of icaritin on autophagy in OSCC cells. As distinct self-destructive processes, autophagy and apoptosis have a very complex interaction. Although, in some cases, autophagy may help to induce apoptosis or necrosis, and could even promote cell death itself. Most studies support the survival role of autophagy in chemotherapy-induced cell death, suppressing apoptosis [41,42]. Therefore, the interference of the interplay between autophagy and apoptosis has an important role in drug treatment of cancer. Icaritin could induce autophagy and apoptosis via STAT3 signaling inhibition in OSCC. Some studies demonstrated that suppressing autophagy can enhance the efficacy of blocking STAT3 signaling on the apoptosis of OSCC cells [20,40]. Thus, we speculated that combining icaritin with autophagy inhibitor may improve the therapeutic efficiency for cancer, which is worth further exploration in the future.

Oral cancers are often associated with stepwise accumulation of genetic and epigenetic alterations, generally triggered by exposure to chronic tobacco use or betel nut chewing [34]. During this multistep carcinogenesis, premalignant lesions are associated with a likely progression to cancer [6]. As the rate of malignant conversion of oral leukoplakia is $0.13 \%-34 \%$, it would be suitable for studying chemopreventive agents by targeting these lesions $[5,43]$. Previous studies showed that oral administration of 4-NQO could induce the formation of dysplastic lesions and OSCC in mice $[33,44]$. In addition, the histological and molecular changes during OSCC carcinogenesis in this model were similar to human OSCC [33]. In the present study, we used this model to mimic the process of oral carcinogenesis in humans. Our results showed that the number of malignant tumors in the treated group was significantly less compared with the control group. Thus, icaritin could suppress the progression of oral epithelial dysplasias into carcinomas. Grandis et al. found that the incidence of total preneoplastic and neoplastic lesions significantly decreased due to erlotinib treatment at the beginning of 4NQO exposure [33]. In the present study, the total number of dysplasia also decreased after icaritin treatment, although a statistical difference was not observed between icaritin-treated group and control group, which might be due to the short icaritin time of exposure. This evidence suggested that icaritin efficacy could be improved through its long-term use. More importantly, as a natural product, icaritin showed few adverse reactions such as body weight loss in vivo. Taken together, icaritin may become an attractive chemopreventive agent to hamper the progression of premalignant lesions converting into OSCC.

\section{Materials and Methods}

\subsection{Proliferation Assay}

Human OSCC cell lines CAL27 and SCC9 were cultured in DMEM or DMEM/F12 (Gibco, Carlsbad, CA, USA) respectively, with 10\% fetal bovine serum (FBS; Gibco). Cells were incubated in a humidified atmosphere of $95 \%$ air and $5 \% \mathrm{CO}_{2}$ at $37{ }^{\circ} \mathrm{C}$. Cells were seeded at a density of $3 \times 10^{3}$ cells per well in 96-well culture plates. After overnight incubation, the cells were treated with either DMSO (vehicle control; Sigma, St. Louis, MO, USA) or icaritin at different concentrations (>98\% pure; Baoji Herbest Bio-Tech Corporation, Baoji, China) $(0,2,4,8$, and $16 \mu \mathrm{M}$ ) for 24, 48, and $72 \mathrm{~h}$. Cell proliferation was then detected using cell counting kit-8 (CCK-8; Dojin Laboratory, Kumamoto, Japan) according to the manufacturer's instructions. Absorbance was measured at $450 \mathrm{~nm}$ using a 96-well multiscanner autoreader (Thermo Fisher Scientific, Waltham, MA, USA). Then, the inhibition efficiency of icaritin and the OSCC cells growth curves were calculated with the same optical density (OD) values in different ways.

\subsection{Apoptosis Assay}

CAL27 cells and SCC9 cells $\left(3 \times 10^{5}\right)$ were seeded in 6-well plate. Next, cells were incubated with icaritin for $48 \mathrm{~h}$ at the concentrations previously mentioned. Floating and attached cells were collected and cell apoptosis was assessed using Annexin V-FITC and PI apoptosis detection kit (BD Biosciences, 
San Diego, CA, USA) by FACSCaliber flow cytometry (Becton-Dickinson, Franklin Lakes, NJ, USA), according to the manufacturer's instructions. Data were analyzed by FlowJo software (Treestar, Ashland, OR, USA).

\subsection{Autophagy Assay}

The increased acidic vesicular organelles (AVOs) are correlated with increased autophagosomes, which could indicate the formation of autophagolysosomes. Monodansylcadaverine (MDC) (Sigma, St. Louis, MO, USA) staining was employed to detect the AVO formation in the autophagic process. CAL27 cells and SCC9 cells were seeded on slides in 24-well plate and incubated with icaritin for $48 \mathrm{~h}$ at the concentrations previously mentioned. CAL27 cell and SCC9 cell autophagy was induced by $1 \mu \mathrm{M}$ rapamycin for $3 \mathrm{~h}$ to obtain the positive control. Autophagic vacuoles of treated cells were stained with $0.05 \mathrm{mM} \mathrm{MDC}$ and incubated at $37^{\circ} \mathrm{C}$ for $30 \mathrm{~min}$. After incubation, cells were washed by phosphate buffered saline (PBS) three times, and observed by a laser scanning confocal fluorescence microscopy (Olympus, Tokyo, Japan).

\subsection{Western Blot}

CAL27 cells and SCC9 cells were treated with icaritin for $48 \mathrm{~h}$ at the concentrations previously mentioned, then were harvested, washed immediately with ice-cold PBS, and lysed by RIPA buffer containing PMSF (Beyotime Biotechnology, Shanghai, China) and phosphatase inhibitors (Beyotime Biotechnology). The whole cell lysates were collected after centrifugation at $12,000 \times g$ for $15 \mathrm{~min}$ at $4{ }^{\circ} \mathrm{C}$, and protein concentration in the supernatants was determined by $\mathrm{BCA}$ assay kit (Beyotime Biotechnology). Equal amounts of protein $(6 \mu \mathrm{g} / \mathrm{lane})$ were loaded onto $12 \%$ SDS-polyacrylamide gels, subjected to electrophoresis, and transferred to PVDF membranes (Millipore, Billerica, MA, USA). After blocking with 5\% nonfat milk for $1 \mathrm{~h}$, membranes were incubated overnight at $4{ }^{\circ} \mathrm{C}$ with primary antibodies against proliferation related proteins cyclin D1 and cyclin A2 (Cell Signaling Technology, Danvers, MA, USA); apoptosis related proteins cleaved-caspase 3 and cleaved-PAPR (Cell Signaling Technology); autophagy related proteins p62/SQSTM1, BECN1, LC3B, and ATG9B (Abcam, Cambridge, MA, USA) and STAT3 associated proteins STAT3, phospho-STAT3, phospho-JAK2, phospho-extracellular signal-regulated kinase 1/2 (p-ERK1/2) (Cell Signaling Technology). After washed three times with TBST, membranes were then incubated with HRP-labelled secondary antibody for $1 \mathrm{~h}$ at room temperature. The protein bands were visualized using ECL reagent. $\beta$-actin was used as the internal loading control. Western blot experiments were repeated at least three times.

\section{5. siRNA Interference for STAT3}

CAL27 cells were seeded onto a culture plate and transiently transfected with human STAT3 siRNA (5'-CCGTGGAACCATACACAAA-3') and negative control siRNA according to the manufacturer's transfection protocol (RiboBio, Guangzhou, China). After $48 \mathrm{~h}$ transfection, cells were treated with the indicated concentration of icaritin for $48 \mathrm{~h}$, and cell viability and apoptosis were measured.

\subsection{Animal Studies}

The animal protocol was approved and supervised by the Ethical Board of the Center for Laboratory Animal Science at the Wuhan University (No. 00133334; 14 April 2015) and conducted according to the Guidelines for the Care and Use of Laboratory Animals. Female C57BL/ 6 mice (eight weeks old) were housed in a temperature-controlled room $\left(25^{\circ} \mathrm{C}\right)$ under $12 \mathrm{~h} / 12 \mathrm{~h}$ light/dark cycle and have access to food and water ad libitum. To explore the OSCC preventing effect of icaritin in vivo, an OSCC mouse model was established. 4-Nitroquinoline-1-oxide (4NQO; Sigma, St. Louis, MO, USA) was added in the drinking water $(50 \mu \mathrm{g} / \mathrm{mL})$. All mice received a full oral examination under anesthesia every week. After 14 weeks, 16 mice with at least one premalignant lesion in the tongue were 
reverted to regular water, and randomly divided into treatment and control groups, which received intraperitoneal (i.p) injections of icaritin $(10 \mathrm{mg} / \mathrm{kg})$ dissolved in $0.3 \%$ sodium carboxymethylcellulose (CMC-Na; Sigma, St. Louis, MO, USA) or an equal amount of solvent once every other day. All animals were euthanized at week 22, and tissues were fixed and embedded in paraffin for histopathological analysis and subsequent immunohistochemical staining.

\subsection{Immunohistochemistry Assay}

Concentrations of primary antibodies were used for monoclonal mouse anti-p-STAT3 1:400 and anti-p-JAK2 1:200, which were purchased from Cell Signaling Technology. Paraffin sections were dewaxed and rehydrated through a series of xylene, graded alcohol, and water immersion steps. The deparaffinized and hydrated slides were treated with $3 \% \mathrm{H}_{2} \mathrm{O}_{2}$ at $37{ }^{\circ} \mathrm{C}$ for $20 \mathrm{~min}$ to block endogenous peroxidase activity and then washed with PBS. Slides were treated with $5 \%$ normal goat serum for $30 \mathrm{~min}$ to block non-specific binding, and incubated with primary antibodies at $4{ }^{\circ} \mathrm{C}$ overnight. After washing with PBS, they were incubated with biotinylated goat anti-mouse or goat anti-rabbit IgG antibody (Zhongshan Golden Bridge Ltd., Beijing, China) for $15 \mathrm{~min}$ and washed again with PBS. DAB detection kit (Zhongshan Golden Bridge Ltd., Beijing, China) was used for staining. The slices were visualized for $1 \mathrm{~min}$ following staining, then re-stained with hematoxylin and sealed. Cells with brown staining were considered positive. The staining intensity and positive ratio of the mentioned proteins were observed under microscope by two independent scorers blinded to the clinical parameters.

\subsection{Statistical Analysis}

Data were expressed as means \pm standard deviation (SD) for the indicated number of separate experiments. $\mathrm{IC}_{50}$ for icaritin was calculated from linear transformation of dose-response curves. The differences between experimental and control groups were analyzed using Student's $t$ test or one-way ANOVA. All statistical analyses were performed using GraphPad Prism version 5.0 (Graphpad Software Inc., La Jolla, CA, USA) and SPSS 17.0 (Chicago, IL, USA). Statistical significance was defined as $p<0.05$.

\section{Conclusions}

Our findings indicated that icaritin inhibited proliferation and induced apoptosis and autophagy in OSCC cells mainly through STAT3 signaling inhibition. Remarkably, icaritin could prevent oral premalignant lesion conversion to squamous cell carcinomas in vivo. Hence, the natural product icaritin could be considered as a promising agent in OSCC treatment and prevention.

Acknowledgments: This work was supported by grants to Gang Zhou from the Nonprofit Industry Research Specific Fund of National Health and Family Planning Commission of China (No. 201502018) and the National Natural Science Foundation of China (Nos. 81170972 and 81371147).

Author Contributions: Gang Zhou and Jian-Guang Yang designed the experiments and wrote the manuscript; Jian-Guang Yang, Rui Lu, and Xiao-Jing Ye performed the experiments; Jing Zhang and Ya-Qin Tan helped analyze the data and correct the manuscript. All authors read and approved the final manuscript.

Conflicts of Interest: The authors declare no conflict of interest.

\section{References}

1. Adams, D. Oral cancer: Early diagnosis. Dent. Today 2014, 33, 8. [PubMed]

2. Torre, L.A.; Bray, F.; Siegel, R.L.; Ferlay, J.; Lortet-Tieulent, J.; Jemal, A. Global cancer statistics, 2012. CA Cancer J. Clin. 2015, 65, 87-108. [CrossRef] [PubMed]

3. Guneri, P.; Epstein, J.B. Late stage diagnosis of oral cancer: Components and possible solutions. Oral Oncol. 2014, 50, 1131-1136. [CrossRef] [PubMed]

4. Kumar, M.; Nanavati, R.; Modi, T.G.; Dobariya, C. Oral cancer: Etiology and risk factors: A review. J. Cancer Res. Ther. 2016, 12, 458-463. [CrossRef] [PubMed] 
5. Boy, S.C. Leukoplakia and erythroplakia of the oral mucosa-A brief overview. SADJ J. S. Afr. Dent. Assoc. 2012, 67, 558-560.

6. Warnakulasuriya, S.; Reibel, J.; Bouquot, J.; Dabelsteen, E. Oral epithelial dysplasia classification systems: Predictive value, utility, weaknesses and scope for improvement. J. Oral Pathol. Med. 2008, 37, 127-133. [CrossRef] [PubMed]

7. Jiang, M.C.; Chen, X.H.; Zhao, X.; Zhang, X.J.; Chen, W.F. Involvement of IGF-1 receptor signaling pathway in the neuroprotective effects of Icaritin against $\mathrm{MPP}^{+}$-induced toxicity in MES23.5 cells. Eur. J. Pharmacol. 2016, 786, 53-59. [CrossRef] [PubMed]

8. Wo, Y.B.; Zhu, D.Y.; Hu, Y.; Wang, Z.Q.; Liu, J.; Lou, Y.J. Reactive oxygen species involved in prenylflavonoids, icariin and icaritin, initiating cardiac differentiation of mouse embryonic stem cells. J. Cell. Biochem. 2008, 103, 1536-1550. [CrossRef] [PubMed]

9. Huang, X.; Zhu, D.; Lou, Y. A novel anticancer agent, icaritin, induced cell growth inhibition, G1 arrest and mitochondrial transmembrane potential drop in human prostate carcinoma PC-3 cells. Eur. J. Pharmacol. 2007, 564, 26-36. [CrossRef] [PubMed]

10. Tong, J.S.; Zhang, Q.H.; Huang, X.; Fu, X.Q.; Qi, S.T.; Wang, Y.P.; Hou, Y.; Sheng, J.; Sun, Q.Y. Icaritin causes sustained ERK1/2 activation and induces apoptosis in human endometrial cancer cells. PLoS ONE 2011, 6, e16781. [CrossRef] [PubMed]

11. Guo, Y.; Zhang, X.; Meng, J.; Wang, Z.Y. An anticancer agent icaritin induces sustained activation of the extracellular signal-regulated kinase (ERK) pathway and inhibits growth of breast cancer cells. Eur. J. Pharmacol. 2011, 658, 114-122. [CrossRef] [PubMed]

12. Li, S.; Priceman, S.J.; Xin, H.; Zhang, W.; Deng, J.; Liu, Y.; Huang, J.; Zhu, W.; Chen, M.; Hu, W.; et al. Icaritin inhibits JAK/STAT3 signaling and growth of renal cell carcinoma. PLoS ONE 2013, 8, e81657. [CrossRef] [PubMed]

13. Zheng, Q.; Liu, W.W.; Li, B.; Chen, H.J.; Zhu, W.S.; Yang, G.X.; Chen, M.J.; He, G.Y. Anticancer effect of icaritin on human lung cancer cells through inducing $S$ phase cell cycle arrest and apoptosis. J. Huazhong Univ. Sci. Technol. Med. Sci. 2014, 34, 497-503. [CrossRef] [PubMed]

14. Zhu, S.; Wang, Z.; Li, Z.; Peng, H.; Luo, Y.; Deng, M.; Li, R.; Dai, C.; Xu, Y.; Liu, S.; et al. Icaritin suppresses multiple myeloma, by inhibiting IL-6/JAK2/STAT3. Oncotarget 2015, 6, 10460-10472. [CrossRef] [PubMed]

15. Li, Q.; Huai, L.; Zhang, C.; Wang, C.; Jia, Y.; Chen, Y.; Yu, P.; Wang, H.; Rao, Q.; Wang, M.; et al. Icaritin induces AML cell apoptosis via the MAPK/ERK and PI3K/AKT signal pathways. Int. J. Hematol. 2013, 97, 617-623. [CrossRef] [PubMed]

16. Zhu, J.; Li, Z.; Zhang, G.; Meng, K.; Kuang, W.; Li, J.; Zhou, X.; Li, R.; Peng, H.; Dai, C.; et al. Icaritin shows potent anti-leukemia activity on chronic myeloid leukemia in vitro and in vivo by regulating MAPK/ERK/JNK and JAK2/STAT3 /AKT signalings. PLoS ONE 2011, 6, e23720. [CrossRef] [PubMed]

17. Xu, B.; Jiang, C.; Han, H.; Liu, H.; Tang, M.; Liu, L.; Ji, W.; Lu, X.; Yang, X.; Zhang, Y.; et al. Icaritin inhibits the invasion and epithelial-to-mesenchymal transition of glioblastoma cells by targeting EMMPRIN via PTEN/AKt/HIF-1alpha signalling. Clin. Exp. Pharmacol. Physiol. 2015, 42, 1296-1307. [CrossRef] [PubMed]

18. Mali, S.B. Review of STAT3 (Signal Transducers and Activators of Transcription) in head and neck cancer. Oral Oncol. 2015, 51, 565-569. [CrossRef] [PubMed]

19. Lai, S.Y.; Johnson, F.M. Defining the role of the JAK-STAT pathway in head and neck and thoracic malignancies: Implications for future therapeutic approaches. Drug Resist. Updates 2010, 13, 67-78. [CrossRef] [PubMed]

20. Fan, T.F.; Bu, L.L.; Wang, W.M.; Ma, S.R.; Liu, J.F.; Deng, W.W.; Mao, L.; Yu, G.T.; Huang, C.F.; Liu, B.; et al. Tumor growth suppression by inhibiting both autophagy and STAT3 signaling in HNSCC. Oncotarget 2015, 6, 43581-43593. [PubMed]

21. Yu, H.; Lee, H.; Herrmann, A.; Buettner, R.; Jove, R. Revisiting STAT3 signalling in cancer: New and unexpected biological functions. Nat. Rev. Cancer 2014, 14, 736-746. [CrossRef] [PubMed]

22. Yu, H.; Pardoll, D.; Jove, R. STATs in cancer inflammation and immunity: A leading role for STAT3. Nat. Rev. Cancer 2009, 9, 798-809. [CrossRef] [PubMed]

23. Shen, S.; Niso-Santano, M.; Adjemian, S.; Takehara, T.; Malik, S.A.; Minoux, H.; Souquere, S.; Marino, G.; Lachkar, S.; Senovilla, L.; et al. Cytoplasmic STAT3 represses autophagy by inhibiting PKR activity. Mol. Cell 2012, 48, 667-680. [CrossRef] [PubMed] 
24. Sen, M.; Pollock, N.I.; Black, J.; DeGrave, K.A.; Wheeler, S.; Freilino, M.L.; Joyce, S.; Lui, V.W.; Zeng, Y.; Chiosea, S.I.; et al. JAK kinase inhibition abrogates STAT3 activation and head and neck squamous cell carcinoma tumor growth. Neoplasia 2015, 17, 256-264. [CrossRef] [PubMed]

25. Peyser, N.D.; Freilino, M.; Wang, L.; Zeng, Y.; Li, H.; Johnson, D.E.; Grandis, J.R. Frequent promoter hypermethylation of PTPRT increases STAT3 activation and sensitivity to STAT3 inhibition in head and neck cancer. Oncogene 2016, 35, 1163-1169. [CrossRef] [PubMed]

26. Bonner, J.A.; Yang, E.S.; Trummell, H.Q.; Nowsheen, S.; Willey, C.D.; Raisch, K.P. Inhibition of STAT-3 results in greater cetuximab sensitivity in head and neck squamous cell carcinoma. Radiother. Oncol. 2011, 99, 339-343. [CrossRef] [PubMed]

27. Sen, M.; Thomas, S.M.; Kim, S.; Yeh, J.I.; Ferris, R.L.; Johnson, J.T.; Duvvuri, U.; Lee, J.; Sahu, N.; Joyce, S.; et al. First-in-human trial of a STAT3 decoy oligonucleotide in head and neck tumors: Implications for cancer therapy. Cancer Discov. 2012, 2, 694-705. [CrossRef] [PubMed]

28. Bendris, N.; Lemmers, B.; Blanchard, J.M. Cell cycle, cytoskeleton dynamics and beyond: The many functions of cyclins and CDK inhibitors. Cell Cycle 2015, 14, 1786-1798. [CrossRef] [PubMed]

29. Galluzzi, L.; Lopez-Soto, A.; Kumar, S.; Kroemer, G. Caspases Connect Cell-Death Signaling to Organismal Homeostasis. Immunity 2016, 44, 221-231. [CrossRef] [PubMed]

30. Agarwal, A.; Mahfouz, R.Z.; Sharma, R.K.; Sarkar, O.; Mangrola, D.; Mathur, P.P. Potential biological role of poly (ADP-ribose) polymerase (PARP) in male gametes. Reprod. Biol. Endocrinol. 2009, 7, 143. [CrossRef] [PubMed]

31. Cui, X.; Liu, J.; Bai, L.; Tian, J.; Zhu, J. Interleukin-6 induces malignant transformation of rat mesenchymal stem cells in association with enhanced signaling of signal transducer and activator of transcription 3. Cancer Sci. 2014, 105, 64-71. [CrossRef] [PubMed]

32. Brown, M.E.; Bear, M.D.; Rosol, T.J.; Premanandan, C.; Kisseberth, W.C.; London, C.A. Characterization of STAT3 expression, signaling and inhibition in feline oral squamous cell carcinoma. BMC Vet. Res. 2015, 11, 206. [CrossRef] [PubMed]

33. Leeman-Neill, R.J.; Seethala, R.R.; Singh, S.V.; Freilino, M.L.; Bednash, J.S.; Thomas, S.M.; Panahandeh, M.C.; Gooding, W.E.; Joyce, S.C.; Lingen, M.W.; et al. Inhibition of EGFR-STAT3 signaling with erlotinib prevents carcinogenesis in a chemically-induced mouse model of oral squamous cell carcinoma. Cancer Prev. Res. 2011, 4, 230-237. [CrossRef] [PubMed]

34. Rahman, M.A.; Amin, A.R.; Shin, D.M. Chemopreventive potential of natural compounds in head and neck cancer. Nutr. Cancer 2010, 62, 973-987. [CrossRef] [PubMed]

35. Bollrath, J.; Phesse, T.J.; von Burstin, V.A.; Putoczki, T.; Bennecke, M.; Bateman, T.; Nebelsiek, T.; Lundgren-May, T.; Canli, O.; Schwitalla, S.; et al. gp130-mediated Stat3 activation in enterocytes regulates cell survival and cell-cycle progression during colitis-associated tumorigenesis. Cancer Cell 2009, 15, 91-102. [CrossRef] [PubMed]

36. Shi, L.; Wang, S.; Zangari, M.; Xu, H.; Cao, T.M.; Xu, C.; Wu, Y.; Xiao, F.; Liu, Y.; Yang, Y.; et al. Over-expression of CKS1B activates both MEK/ERK and JAK/STAT3 signaling pathways and promotes myeloma cell drug-resistance. Oncotarget 2010, 1, 22-33. [CrossRef] [PubMed]

37. Jin, L.; Miao, J.; Liu, Y.; Li, X.; Jie, Y.; Niu, Q.; Han, X. Icaritin induces mitochondrial apoptosis by up-regulating miR-124 in human oral squamous cell carcinoma cells. Biomed. Pharmacother. 2016, 85, 287-295. [CrossRef] [PubMed]

38. Marino, G.; Niso-Santano, M.; Baehrecke, E.H.; Kroemer, G. Self-consumption: The interplay of autophagy and apoptosis. Nat. Rev. Mol. Cell Biol. 2014, 15, 81-94. [CrossRef]

39. Kumar, D.; Shankar, S.; Srivastava, R.K. Rottlerin-induced autophagy leads to the apoptosis in breast cancer stem cells: Molecular mechanisms. Mol. Cancer 2013, 12, 171. [CrossRef] [PubMed]

40. Feng, Y.; Ke, C.; Tang, Q.; Dong, H.; Zheng, X.; Lin, W.; Ke, J.; Huang, J.; Yeung, S.C.; Zhang, H. Metformin promotes autophagy and apoptosis in esophageal squamous cell carcinoma by downregulating Stat3 signaling. Cell Death Dis. 2014, 5, e1088. [CrossRef] [PubMed]

41. Eisenberg-Lerner, A.; Bialik, S.; Simon, H.U.; Kimchi, A. Life and death partners: Apoptosis, autophagy and the cross-talk between them. Cell Death Differ. 2009, 16, 966-975. [CrossRef] [PubMed]

42. Song, P.; Ye, L.; Fan, J.; Li, Y.; Zeng, X.; Wang, Z.; Wang, S.; Zhang, G.; Yang, P.; Cao, Z.; et al. Asparaginase induces apoptosis and cytoprotective autophagy in chronic myeloid leukemia cells. Oncotarget 2015, 6, 3861-3873. [CrossRef] [PubMed] 
43. Warnakulasuriya, S.; Ariyawardana, A. Malignant transformation of oral leukoplakia: A systematic review of observational studies. J. Oral Pathol. Med. 2016, 45, 155-166. [CrossRef] [PubMed]

44. Vitale-Cross, L.; Molinolo, A.A.; Martin, D.; Younis, R.H.; Maruyama, T.; Patel, V.; Chen, W.; Schneider, A.; Gutkind, J.S. Metformin prevents the development of oral squamous cell carcinomas from carcinogen-induced premalignant lesions. Cancer Prev. Res. 2012, 5, 562-573. [CrossRef] [PubMed]

(C) 2017 by the authors; licensee MDPI, Basel, Switzerland. This article is an open access article distributed under the terms and conditions of the Creative Commons Attribution (CC-BY) license (http:/ / creativecommons.org/licenses/by/4.0/). 